\title{
Associations between clinical canine leishmaniosis and multiple vector-borne co- infections: a case-control serological study
}

\author{
Charalampos Attipa ${ }^{1,2,3,4^{*}}$ (D) Laia Solano-Gallego ${ }^{5}$, Christian M. Leutenegger ${ }^{6}$, Kostas Papasouliotis ${ }^{1,7}$, \\ Francesca Soutter ${ }^{2}$, Jörg Balzer ${ }^{8}$, Scott Carver ${ }^{9}$, Jesse S. Buch ${ }^{10}$ and Séverine Tasker ${ }^{1,11,12}$
}

\begin{abstract}
Background: Dogs that have clinical leishmaniosis (ClinL), caused by the parasite Leishmania infantum, are commonly co-infected with other pathogens, especially vector-borne pathogens (VBP). A recent PCR-based study found that ClinL dogs are more likely to be additionally infected with the rickettsial bacteria Ehrlichia canis. Further information on coinfections in ClinL cases with VBP, as assessed by serology, is required. The research described in this report determined if dogs with ClinL are at higher risk of exposure to VBP than healthy control dogs using a case-control serology study.

Results: Of the 47 dogs with ClinL, anti-E. canis/ Ehrlichia ewingii antibodies were detected in 17 (36.2\%), antiAnaplasma phagocytophilum/Anaplasma platys antibodies in $5(10.6 \%)$ and antigen for Dirofilaria immitis in 2 (4.3\%). Of the 87 control dogs, anti-E. canis/E. ewingii antibodies were detected in 14 (16.1\%) and anti-A. phagocytophilum/ A. platys antibodies in 2 (2.3\%). No anti-Borrelia burgdorferi antibody tests were positive. No statistical differences between the ClinL dogs and control dogs regarding lifestyle or use of ectoparasitic prevention, were identified. The ClinL was significantly associated with anti-E. canis/E. ewingii antibodies (odds ratio $=2.9$, 95\% confidence interval: $1.3-$ $6.7, P=0.010)$ compared to controls by both multivariable logistic regression and structural equation modelling.

Conclusions: It was demonstrated that an increased risk for E. canis/E. ewingii seropositivity is present in dogs with ClinL compared to clinically healthy control dogs, despite similar ectoparasitic prevention use and lifestyle. Based on these findings it is suggested that dogs with ClinL should not only be tested for $E$. canis co-infection using PCR but also serologically for E. canis/E. ewingii.
\end{abstract}

Keywords: Dog, Leishmania infantum, Ehrlichia canis, Borrelia burgdorferi, Acanthocheilonema reconditum, Vectorborne pathogen, Co-infection, Cyprus

\section{Background}

Canine leishmaniosis (CanL) is a significant zoonotic disease in Mediterranean region and is caused by the kinetoplastid parasite Leishmania infantum that is transmitted by sand flies vectors belonging to the Phlebotomus genus [1]. Often vector-borne pathogens (VBP) such as Anaplasma platys, Ehrlichia canis, Dirofilaria immitis, Hepatozoon canis and Babesia vogeli concurrently infect dogs which have clinical leishmaniosis

\footnotetext{
* Correspondence: attipacy@gmail.com

'Molecular Diagnostic Unit, Diagnostic Laboratories, Bristol Veterinary School and Langford Vets, University of Bristol, Langford, UK

${ }^{2}$ Department of Pathobiology and Population Sciences, The Royal Veterinary College, University of London, Hatfield, Hertfordshire, UK

Full list of author information is available at the end of the article
}

(ClinL) despite being transmitted by vectors different than these for L. infantum [2-4]. Such co-infections can result in an unexpected incubation time, atypical clinical sings, more severe clinicopathological abnormalities and worse prognosis for the dogs with CanL, compared with dogs that have CanL alone [2, 3, 5]. Furthermore, a recent PCR-based case-control study found that dogs with ClinL are in higher risk to be co-infected with $E$. canis compared to healthy matched controls [6]. Additional information on co-infections in ClinL cases with VBP, as assessed by serology in case-control studies, is required.

The aim of this study was to examine if dogs with ClinL are more likely to be exposed to A. phagocytophilum/

(c) The Author(s). 2019 Open Access This article is distributed under the terms of the Creative Commons Attribution 4.0 International License (http://creativecommons.org/licenses/by/4.0/), which permits unrestricted use, distribution, and reproduction in any medium, provided you give appropriate credit to the original author(s) and the source, provide a link to the Creative Commons license, and indicate if changes were made. The Creative Commons Public Domain Dedication waiver (http://creativecommons.org/publicdomain/zero/1.0/) applies to the data made available in this article, unless otherwise stated. 
A. platys, B. burgdorferi and E. canis/Ehrlichia ewingii, or infected for $D$. immitis than clinically healthy controls.

\section{Results}

Serum was available in 47 dogs with ClinL and 87 dog controls that were included in this study. The age of these 134 dogs ranged from 1 up to 12 years (median 4 years, interquartile range 3 years) and 98 (73\%) were pedigree including Cocker spaniel, Segugio Italiano, Beagle, German Shepherd and other breeds (Additional file 1).

In the ClinL group, anti-A. phagocytophilum/A. platys antibodies were detected in $5(10.6 \%)$, anti-E. canis/E. ewingii antibodies in $17(36.2 \%)$ and antigen for $D$. immitis in $2(4.3 \%)$ dogs. Of the 87 control dogs, anti-A. phagocytophilum/A. platys antibodies were detected in 2 $(2.3 \%)$ and anti-E. canis/E. ewingii antibodies in 14 (16.1\%). No anti-B. burgdorferi antibody tests were positive (Fig. 1). Table 1 summarizes the demographic characteristics and the serology findings. The two dogs with $D$. immitis antigens underwent microfilaria PCR specification which was positive for $A$. reconditum and negative for $D$. immitis for both cases.

ClinL was significantly associated with anti-E. canis/E. ewingii antibodies [odds ratio $(\mathrm{OR})=2.9,95 \%$ confidence interval (CI): 1.3-6.7, $P=0.010]$, compared to healthy controls using multivariable logistic regression. The presence of anti-A. phagocytophilum/A. platys antibodies was initially associated significantly with ClinL compared to controls using univariable analysis $(\mathrm{OR}=$ 5.1, 95\% CI: $0.9-27.2, P=0.038)$ but this association was not maintained during multivariable logistic regression
Table 1 Demographic characteristics of the study dog groups and serology results for the VBPs tested. All dogs tested negative for Borrelia burgdorferi antibodies

\begin{tabular}{|c|c|c|}
\hline Characteristic & $\begin{array}{l}\text { No. of cases } \\
\text { ClinL }(\%)(n=47)\end{array}$ & $\begin{array}{l}\text { No. of Control } \\
(\%)(n=87)\end{array}$ \\
\hline \multicolumn{3}{|l|}{ Age in years } \\
\hline Median & 3.0 & 4.1 \\
\hline Interquartile range & 3.3 & 3.0 \\
\hline \multicolumn{3}{|l|}{ Sex } \\
\hline Male & $23(49)$ & $47(54)$ \\
\hline Female & $24(51)$ & $40(46)$ \\
\hline \multicolumn{3}{|l|}{ Lifestyle } \\
\hline Outdoors & $34(72)$ & $65(75)$ \\
\hline Mainly indoors & $13(28)$ & $22(25)$ \\
\hline \multicolumn{3}{|c|}{ Ectoparasitic prevention } \\
\hline Used & $16(34)$ & $36(41)$ \\
\hline Not used & $31(66)$ & $51(59)$ \\
\hline \multicolumn{3}{|l|}{ Breed } \\
\hline Pedigree & $33(70)$ & $65(75)$ \\
\hline Crossbreed & $14(30)$ & $22(25)$ \\
\hline \multicolumn{3}{|l|}{ E. canis/E. ewingii } \\
\hline Positive & $17(36)$ & $14(16)$ \\
\hline Negative & $30(64)$ & $73(84)$ \\
\hline \multicolumn{3}{|c|}{ A. phagocytophilum/A. platys } \\
\hline Positive & $5(10)$ & $2(2)$ \\
\hline Negative & $42(90)$ & $85(98)$ \\
\hline \multicolumn{3}{|l|}{ D. immitis } \\
\hline Positive & $2(4)$ & $0(0)$ \\
\hline Negative & $45(96)$ & $87(100)$ \\
\hline
\end{tabular}

Abbreviations: VBP Vector-borne pathogen, ClinL Clinical leishmaniosis, E. canis Ehrlichia canis, E. ewingii Ehrlichia ewingii, D. immitis Dirofilaria immitis, A. phagocytophilum Anaplasma phagocytophilum, A. platys. Anaplasma platys

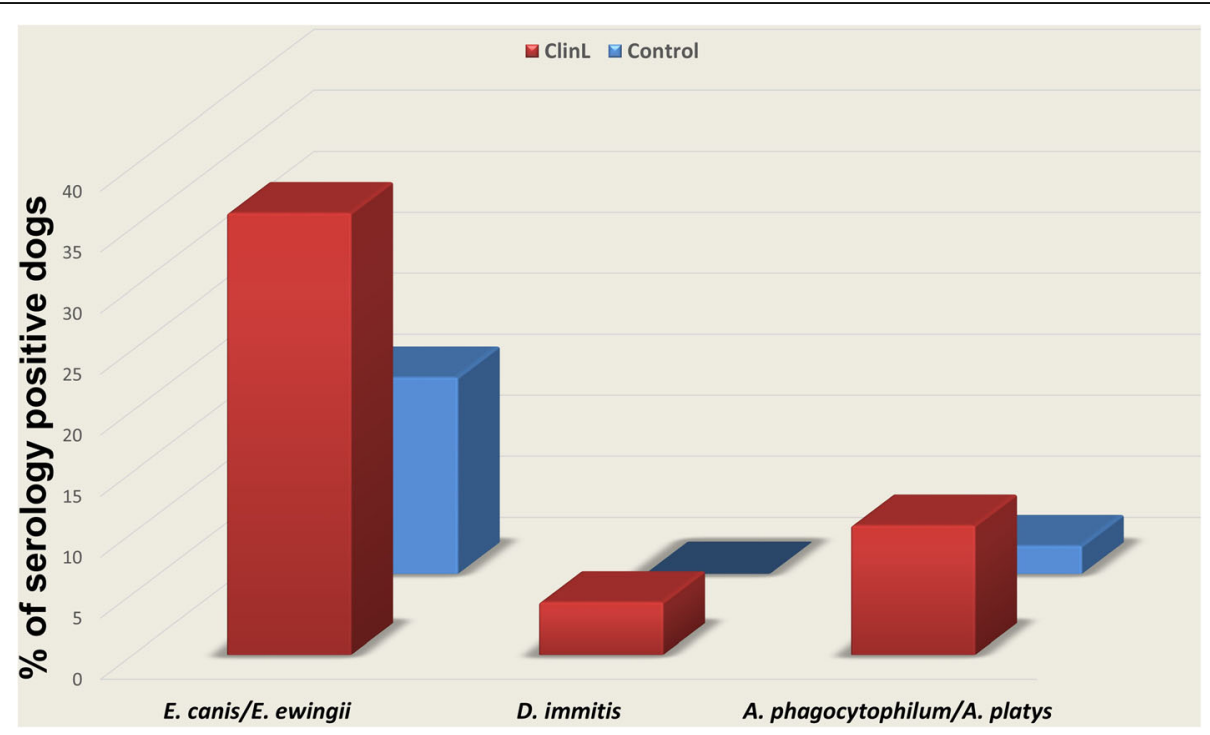

Fig. 1 Comparison of VBP percentages detected by serology between dogs with ClinL $(n=47)$ and healthy control dogs $(n=87)$. Abbreviations: VBP, vector-borne pathogen; ClinL, clinical leishmaniosis; E. canis, Ehrlichia canis; E. ewingii, Ehrlichia ewingii; D. immitis, Dirofilaria immitis; A. phagocytophilum, Anaplasma phagocytophilum; A. platys. Anaplasma platys 
analysis. The numbers of $D$. immitis were very low hindering any further statistical analysis. Age, breed, sex, lifestyle, and use of ectoparasitic prevention were not statistically different between the ClinL and the control dogs.

Two associations were identified based on SEM (Fig. 2, Table 2). It was more likely for dogs with ClinL be $E$. canis/E. ewingii seropositive and dogs seropositive for $E$. canis/E. ewingii are more likely to have be infected with E. canis based on PCR. A trend was identified between dogs with ClinL and $A$. phagocytophilum/A. platys seropositive.

\section{Discussion}

The findings from this serology study are in agreement with previous studies [3, 7] and further support the findings from the initial PCR based study, using a fairly similar cohort of samples, in which it was demonstrated that it is 12 times more likely for dogs with ClinL be co-infected with $E$. canis compared with healthy canine controls (CI: 1.5-106.0, $P=0.022$ ) [6]. A previous 3-year longitudinal study, evaluating $E$. canis and $L$. infantum co-infection in naturally exposed dogs, found that $E$. canis infection preceded L. infantum infection in dogs with dual infections, thus suggesting that $E$. canis could contribute in the establishment of ClinL [7]. Interestingly, a recent study by Baxarias et al. [5] from Catalonia (Spain) found that dogs with ClinL were four times more likely to be seropositive for Rickettsia conorii and 14 times most likely to be seropositive for A. phagocytophilum compared with healthy controls, but they did not found an association between ClinL and E. canis seroreactivity. This discrepancy probably reflects the different prevalence of these pathogens in Cyprus and other Mediterranean areas in comparison to Catalonia.

The seroprevalence of the various VBP in this specific canine population of 134 dogs from the area of Paphos, Cyprus, revealed a strikingly high seroreactivity to $E$. canis/E. ewingii (23\%) and anti-A. phagocytophilum/A. platys (13\%) antibodies compared to other studies from Mediterranean countries using a similar in-house ELISA kit as the one utilised in this study [8-10]. If quantitative ELISA or IFAT with higher sensitivity, compared to the in-house kit, were used in this study, then seroprevalences of the VBP could have been even higher than these reported [11]. The area of Paphos, Cyprus, may be Lyme disease free as no anti- $B$. burgdorferi antibodies were detected in any of the dogs tested in this study, and the tick vectors that transmit this pathogen, including Ixodes Ricinus, have not yet been identified in Cyprus [12]. In two dogs (1\%) antigens for D. immitis were detected but PCR failed to confirm this infection and instead an infection with $A$. reconditum was identified for both cases. These results may indicate that the dogs had dual infection with both $D$. immitis and $A$. reconditum, and the negative PCR for $D$. immitis was as a result of low level microfilaraemia. However, false positive $D$. immitis results cannot be ruled out entirely especially in light of a recent study from Cyprus in which, using a modified Knott's testing for

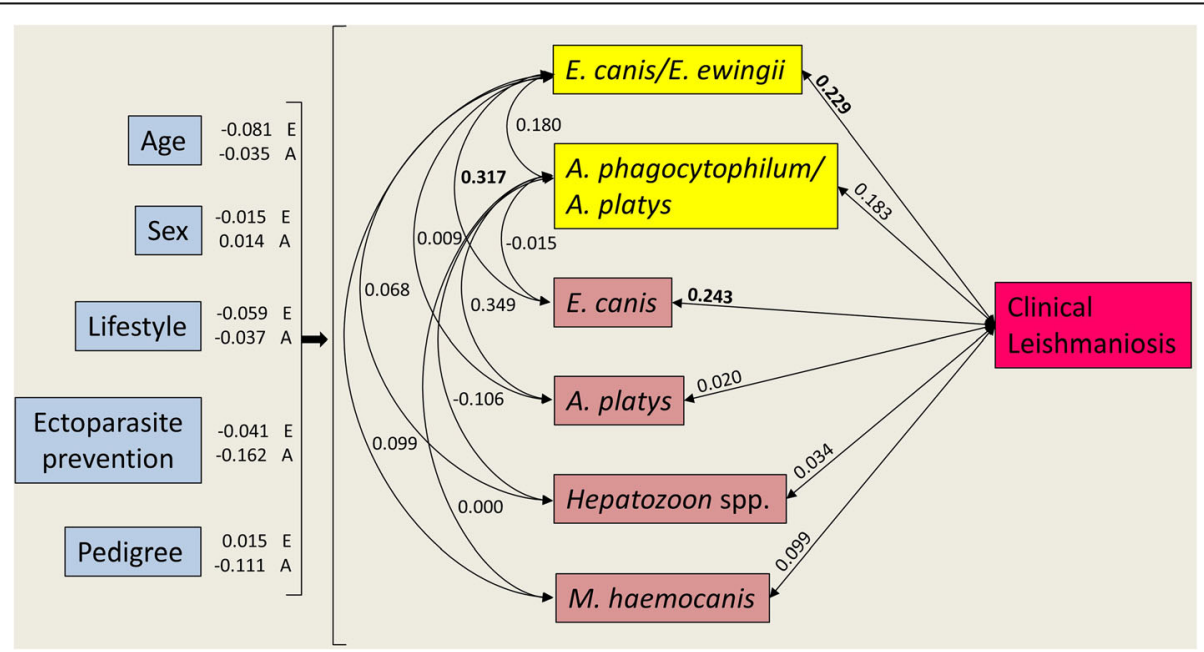

Fig. 2 Structural equation model showing predictors of vector-borne serological exposure status (except ClinL), and pathogen covariance (including ClinL), in domestic dogs. Values represent standardised coefficients among variables. Single headed arrows represent directional/causal relationships and double headed arrows covariance relationships among pathogens. For image clarity the serological status is in yellow boxes and the coefficients of host characteristics predicting pathogens are listed next to each host characteristic. The covariances $E$. canis, A. platys, Hepatozoon spp. and M. haemocanis were PCR-based diagnosed. In all cases, except age, variables are binomial $(0$ or 1$)$ with 1 equal to male, outside, ectoparasitic prevention use, pedigree and positive pathogen status. Standardised coefficients with significant relationships of $P \leq 0.05$ (also see Table 2) are denoted in bold. Abbreviations: ClinL, clinical leishmaniosis; E. canis, Ehrlichia canis; E. ewingii, Ehrlichia ewingii; A. phagocytophilum, Anaplasma phagocytophilum; A. platys. Anaplasma platys; M. haemocanis, Mycoplasma haemocanis 
Table 2 Structural equation model statistical output showing host characteristics predicting serological exposure status for coinfecting pathogens (except ClinL), and the covariance among pathogens (including ClinL), in domestic dogs. The covariances $E$. canis, A. platys, Hepatozoon spp. and M. haemocanis were PCR based diagnosed. In all cases, except age, variables are binomial (0 or 1) with 1 equal to male, outside, ectoparasites controlled, pedigree and positive pathogen status

\begin{tabular}{|c|c|c|c|}
\hline & Standardised coefficient/covariance & $z$-value & $P$-value \\
\hline \multicolumn{4}{|l|}{ E. canis/E. ewingii serology } \\
\hline Age & -0.081 & -0.908 & 0.364 \\
\hline Sex & -0.015 & -0.143 & 0.886 \\
\hline Lifestyle & 0.059 & 0.515 & 0.606 \\
\hline Ectoparasite prevention & -0.041 & -0.336 & 0.737 \\
\hline Pedigree & 0.015 & 0.142 & 0.887 \\
\hline \multicolumn{4}{|l|}{ A. phagocytophilum/A. platys serology } \\
\hline Age & -0.035 & -0.415 & 0.678 \\
\hline Sex & 0.014 & 0.103 & 0.918 \\
\hline Lifestyle & -0.037 & -0.363 & 0.717 \\
\hline Ectoparasite prevention & -0.162 & -1.532 & 0.126 \\
\hline Pedigree & -0.111 & -0.861 & 0.389 \\
\hline \multicolumn{4}{|l|}{ Covariances } \\
\hline E. canis/E. ewingii ClinL & 0.229 & 2.453 & 0.014 \\
\hline A. phagocytophilum/A. platys ClinL & 0.183 & 1.654 & $0.098^{*}$ \\
\hline E. canis/E. ewingii A. phagocytophilum/A. platys & 0.180 & 1.443 & 0.149 \\
\hline E. canis/E. ewingii $\sim$ E. canis & 0.317 & 2.164 & 0.030 \\
\hline E. canis/E. ewingii $\sim$ A. platys & 0.009 & 0.092 & 0.926 \\
\hline E. canis/E. ewingii Hepatozoon spp. & 0.068 & 0.747 & 0.455 \\
\hline E. canis/E. ewingii M. haemocanis & 0.099 & 1.015 & 0.310 \\
\hline A. phagocytophilum/A. platys $\sim$ E. canis & -0.015 & -0.358 & 0.721 \\
\hline A. phagocytophilum/A. platys A. platys & 0.349 & 1.290 & 0.197 \\
\hline A. phagocytophilum/A. platys Hepatozoon spp. & -0.106 & -1.167 & 0.243 \\
\hline A. phagocytophilum/A. platys $\sim$ M. haemocanis & 0.000 & -0.005 & 0.996 \\
\hline
\end{tabular}

Significant relationships $(P<0.05)$ denoted by bold font and trending relationships $(P<0.1)$ denoted by *

Abbreviations: VBP Vector-borne pathogen, ClinL Clinical leishmaniosis, E. canis Ehrlichia canis, E. ewingii Ehrlichia ewingii, A. phagocytophilum Anaplasma phagocytophilum, A. platys. Anaplasma platys, M. haemocanis Mycoplasma haemocanis

morphological identification of microfilariae in a total of 200 healthy dogs which did not receive any kind of heartworm prevention, only $A$. reconditum was identified in 9 dogs (4.5\%) and no D. immitis was found [13].

\section{Conclusions}

It was demonstrated that dogs with ClinL are three times more likely to be exposed to $E$. canis/E. ewingii than clinically healthy control dogs in Paphos, Cyprus. Furthermore, dogs from this area have a high seroreactivity to E. canis/E. ewingii and A. phagocytophilum/A. platys while they are B. burgdorferi free.

\section{Methods}

\section{Study design, site and populations}

The samples used for this serology study were collected under the frame of a previous case-control study design [6]. All samples were collected from canine clinical cases presented to a small animal veterinary hospital in $\mathrm{Pa}$ phos, Cyprus from April 2013 until March 2014. That area was selected since there are high numbers of CanL [14] and various canine VBP have been reported $[15,16]$.

The exact recruiting criteria and the demographic characteristics recorded can be found in the previously published study [6]. Briefly, the dogs that had ClinL were naturally infected and matched with clinically healthy control dogs in-terms of breed, sex, age, living in the same geographical area as well as ideally lifestyle and ectoparasitic prevention use.

\section{Laboratory tests}

Approximately $1-2 \mathrm{ml}$ of surplus serum collected in plain tubes and stored at $-20^{\circ} \mathrm{C}$ until laboratory processing at the Diagnostic Laboratories of the Royal Veterinary College, London, UK. 
A commercial in-clinic patient-side $\mathrm{SNAP}^{\circ} 4 \mathrm{Dx}^{\circ}$ Plus test kit (IDEXX Laboratories, Inc., Westbrook, Maine, USA) was used for the simultaneous detection of antibodies against E. canis/E. ewingii, A. phagocytophilum/A. platys, and $B$. burgdorferi, as well as antigens for $D$. immitis, following manufacturer's instructions. This ELISA kit utilises bi-directional flow of sample and automatic, sequential flow of wash solution and enzyme substrate. For E. canis it detects antibodies to the proteins p30 and p30-1, and for E. ewingii antibodies for p28 protein. For A. phagocytophilum/A. platys the assay detects antibodies against a peptide from the MSP2/p44 major surface protein and the $\mathrm{C}_{6}$ peptide is used for the detection of antibodies to a surface lipoprotein of $B$. burgdorferi. The assay detects antigens produced primarily from the uterus of female $D$. immitis (IDEXX Laboratories, Inc.).

Blood extracted DNA was submitted to IDEXX Laboratories, Ludwigsburg, Germany from all the cases that yielded positive antigens for $D$. immitis for further microfilaria specification using PCR specific assays for D. immitis, Dirofilaria repens, Acanthocheilonema reconditum and Acanthocheilonema dracunculoides. Additionally all samples underwent $L$. infantum serology [17], qPCRs for Leishmania spp. [18], Babesia spp. [19], "Candidatus Mycoplasma haematoparvum" and Mycoplasma haemocanis [20] as well as conventional PCR assays for Ehrlichia/Anaplasma spp. [21] and Hepatozoon spp. [22] under the framework of a previously published study [6].

\section{Data analysis}

The sample size was previously calculated [6] and analyses were performed using SPSS for Windows (version 25.0; SPSS Inc., Chicago IL, USA). A univariable analysis was initially performed to see how each of the explanatory variables was associated with ClinL using Pearson's Chisquare test for categorical explanatory variables (breed, sex, lifestyle, ectoparasitic prevention, positivity for $A$. phagocytophilum/A. platys, positivity for B. burgdorferi, positivity for E. canis/E. ewingii, and positivity for $D$. immitis), and the two-sample t-test or Mann-Whitney $\mathrm{U}$ test for continuous variables (age). Any variables that showed a trend towards significant association with ClinL $(P$-value $<0.1)$ were selected for entry into a multivariable logistic regression. A stepwise selection procedure was used to determine the final model (criteria for entry $P$ value $\leq 0.05$ and for removal $P$-value $>0.1)$.

Additionally, structural equation modelling (SEM) was performed reflecting the hypothesise mechanisms that may be associated with ClinL and other VBP exposure statuses in dogs: (a) causal effects of host characteristics, and (b) pathogen interrelationships, using a method previously described [6].

\section{Supplementary information}

Supplementary information accompanies this paper at https://doi.org/10. 1186/s12917-019-2083-6.

Additional file 1: Table S1. Raw datasets with signalment and test results per case enrolled.

\section{Abbreviations}

Cl: Confidence interval; ClinL: Clinical leishmaniosis; ELISA: Enzyme-linked immunosorbent assay; IFAT: Immunofluorescence antibody test; OR: Odds ratio; QPCR: Quantitative polymerase chain reaction; SEM: Structural equation modelling; VBP: Vector-borne pathogen

\section{Acknowledgements}

Authors would like to thank the veterinarians Dr. Christos Yiapanis and Dr. Kyriaki Neofytou from Cyvets Veterinary Centre, Paphos, Cyprus, for aiding in the collection of blood samples.

\section{Authors' contributions}

CA, LSG, KP, JSB and ST conceived the study, and all participated in its design and coordinated the experiments. CA, LSG and FS designed and performed the collection of the samples. CA performed the serology analysis and the PCR analysis was performed by JB and CML. Statistical analysis was performed by CA, and SC performed the SEM. CA and ST wrote the manuscript with input from all of the authors. All authors reviewed and approved the final manuscript.

\section{Funding}

This clinical study has been sponsored by IDEXX Laboratories, Inc. by providing the SNAP ${ }^{\oplus}$ 4DX Plus ${ }^{\circledast}$ test kit as well as a clinical research grant from Langford Vets, University of Bristol which funded the PCR testing for Babesia spp., "Candidatus Mycoplasma haematoparvum", Mycoplasma haemocanis, Ehrlichia/Anaplasma spp. and Hepatozoon spp. Part of this work done by FS was supported by a Biotechnology and Biological Sciences Research Council Collaborative Awards in Science and Engineering studentship (BB/1015655/1) in partnership with Zoetis and included Leishmania spp. PCR and serology. CA is a Wellcome Trust Clinical Ph.D. Fellow (203919/Z/16/Z) and the open access publication fees have been sponsored by Wellcome Trust.

\section{Availability of data and materials}

The datasets supporting the conclusion of this article are included within the article and Additional file 1.

\section{Ethics approval and consent to participate}

This study was ethically approved by the University of Bristol's Animal Welfare and Ethical Review Board (Veterinary Investigation number: 15/022) as well as the Royal Veterinary Collage's Ethics and Welfare Committee (Veterinary Investigation number: 20141292). All procedures were performed in accordance with Cypriot legislation, which does not requires specific ethical approval from a local ethics committee [The Dogs LAW, N. 184 (I)/ 2002], following written and informed consent being obtained from all dog owners.

\section{Consent for publication}

Not applicable.

\section{Competing interests}

CML, KP, JB and JSB work for IDEXX laboratories the manufacturer of SNAP ${ }^{\circledR}$ 4Dx Plus ${ }^{\circledast}$ test kit.

\section{Author details}

${ }^{1}$ Molecular Diagnostic Unit, Diagnostic Laboratories, Bristol Veterinary School and Langford Vets, University of Bristol, Langford, UK. ${ }^{2}$ Department of Pathobiology and Population Sciences, The Royal Veterinary College, University of London, Hatfield, Hertfordshire, UK. ${ }^{3}$ Cyvets Veterinary Center, Paphos, Cyprus. ${ }^{4}$ Department of Clinical Infection, Microbiology and Immunology, Institute of Infection and Global Health, University of Liverpool, Liverpool, UK. ${ }^{5}$ Departament de Medicina i Cirurgia Animals, Facultat de Veterinària, Universitat Autònoma de Barcelona, Barcelona, Spain. ${ }^{6}$ IDEXX 
Laboratories, Inc., West Sacramento, CA, USA. ${ }^{7}$ Present Address: IDEXX Laboratories Ltd., Wetherby, UK. ${ }^{8} \mathrm{IDEXX} \mathrm{GmbH}$, Ludwigsburg, Germany. ${ }^{9}$ Department of Biological Sciences, University of Tasmania, Hobart, TAS Australia. ${ }^{10}$ IDEXX Laboratories, Inc., Westbrook, ME, USA. ${ }^{11}$ Bristol Veterinary School, University of Bristol, Langford, UK. ${ }^{12}$ The Linnaeus Group, Shirley, UK

Received: 9 February 2019 Accepted: 6 September 2019

Published online: 18 September 2019

\section{References}

1. Killick-Kendrick R. The biology and control of Phlebotomine sand flies. Clin Dermatol. 1999;17(3):279-89.

2. Otranto D, Dantas-Torres F, Breitschwerdt EB. Managing canine vectorborne diseases of zoonotic concern: part one. Trends Parasitol. 2009;25(4): 157-63.

3. De Tommasi AS, Otranto D, Dantas-Torres F, Capelli G, Breitschwerdt EB, de Caprariis D. Are vector-borne pathogen co-infections complicating the clinical presentation in dogs? Parasit Vectors. 2013;6(1):97.

4. Day MJ. The immunopathology of canine vector-borne diseases. Parasit Vectors. 2011:4:48

5. Baxarias $M$, Álvarez-Fernández $A$, Martínez-Orellana $P$, Montserrat-Sangrà $S$, Ordeix $L$, Rojas A, et al. Does co-infection with vector-borne pathogens play a role in clinical canine leishmaniosis? Parasit Vectors. 2018;11(1):135.

6. Attipa C, Solano-Gallego L, Papasouliotis K, Soutter F, Morris D, Helps C, et al. Association between canine leishmaniosis and Ehrlichia canis coinfection: a prospective case-control study. Parasit Vectors. 2018;11(1):184.

7. Mekuzas Y, Gradoni L, Oliva G, Foglia Manzillo V, Baneth G. Ehrlichia canis and Leishmania infantum co-infection: a 3-year longitudinal study in naturally exposed dogs. Clin Microbiol Infect. 2009;15(Suppl 2):30-1.

8. Solano-Gallego L, Llull J, Osso M, Hegarty B, Breitschwerdt E. A serological study of exposure to arthropod-borne pathogens in dogs from northeastern Spain. Vet Res. 2006;37(2):231-44

9. Pantchev N, Schaper R, Limousin S, Norden N, Weise M, Lorentzen L. Occurrence of Dirofilaria immitis and tick-borne infections caused by Anaplasma phagocytophilum, Borrelia burgdorferi sensu lato and Ehrlichia canis in domestic dogs in France: results of a countrywide serologic survey. Parasitol Res. 2009;105(Suppl 1):S101-14

10. Miró G, Montoya A, Roura X, Gálvez R, Sainz A. Seropositivity rates for agents of canine vector-borne diseases in Spain: a multicentre study. Parasit Vectors. 2013;6:117.

11. Bélanger M, Sorenson HL, France MK, Bowie MV, Barbet AF, Breitschwerdt EB, et al. Comparison of serological detection methods for diagnosis of Ehrlichia canis infections in dogs. J Clin Microbiol. 2002;40(9):3506-8.

12. Tsatsaris A, Chochlakis D, Papadopoulos B, Petsa A, Georgalis L, Angelakis E, et al. Species composition, distribution, ecological preference and host association of ticks in Cyprus. Exp Appl Acarol. 2016;70(4):523-42.

13. Kokkinos P, Dimzas D, Pantchev N, Tamvakis A, Balzer J, Diakou A. Filarial infections in dogs in Cyprus, an apparently heartworm free island. Vet Parasitol. 2019;18:100330.

14. Mazeris A, Soteriadou K, Dedet JP, Haralambous C, Tsatsaris A, Moschandreas J, et al. Leishmaniases and the Cyprus paradox. Am J Trop Med Hyg. 2010;82(3):441-8.

15. Attipa C, Hicks CA, Barker EN, Christodoulou V, Neofytou K, Mylonakis ME, et al. Canine tick-borne pathogens in Cyprus and a unique canine case of multiple co-infections. Ticks Tick-Borne Dis. 2017;8(3):341-6.

16. Bouzouraa T, Rene-Martellet M, Chene J, Attipa C, Lebert I, Chalvet-Monfray $\mathrm{K}$, et al. Clinical and laboratory features of canine Anaplasma platys infection in 32 naturally infected dogs in the Mediterranean basin. Ticks Tick-Borne Dis. 2016:7(6):1256-64

17. Solano-Gallego L, Villanueva-Saz S, Carbonell M, Trotta M, Furlanello T, Natale A. Serological diagnosis of canine leishmaniosis: comparison of three commercial ELISA tests (Leiscan, ID screen and Leishmania 96), a rapid test (speed Leish K) and an in-house IFAT. Parasit Vectors. 2014;7:111.

18. Shaw SE, Langton DA, Hillman TJ. Canine leishmaniosis in the United Kingdom: a zoonotic disease waiting for a vector? Vet Parasitol. 2009;163(4): 281-5.

19. Davies S, Abdullah S, Helps C, Tasker S, Newbury H, Wall R. Prevalence of ticks and tick-borne pathogens: Babesia and Borrelia species in ticks infesting cats of Great Britain. Vet Parasitol. 2017;244:129-35.

20. Barker EN, Tasker S, Day MJ, Warman SM, Woolley K, Birtles R, et al. Development and use of real-time PCR to detect and quantify Mycoplasma haemocanis and "Candidatus mycoplasma haematoparvum" in dogs. Vet Microbiol. 2010;140(1-2):167-70.

21. Parola P, Roux V, Camicas J-L, Baradji I, Brouqui P, Raoult D. Detection of ehrlichiae in African ticks by polymerase chain reaction. Trans R Soc Trop Med Hyg. 2000;94(6):707-8.

22. Attipa C, Papasouliotis K, Solano-Gallego L, Baneth G, Nachum-Biala Y,

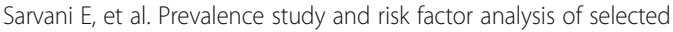
bacterial, protozoal and viral, including vector-borne, pathogens in cats from Cyprus. Parasit Vectors. 2017;10(1):130.

\section{Publisher's Note}

Springer Nature remains neutral with regard to jurisdictional claims in published maps and institutional affiliations.
Ready to submit your research? Choose BMC and benefit from:

- fast, convenient online submission

- thorough peer review by experienced researchers in your field

- rapid publication on acceptance

- support for research data, including large and complex data types

- gold Open Access which fosters wider collaboration and increased citations

- maximum visibility for your research: over $100 \mathrm{M}$ website views per year

At BMC, research is always in progress.

Learn more biomedcentral.com/submissions 